\title{
EI PRONAFCAP y su práctica pedagógica, en las instituciones educativas beneficiarias, región Puno
}

\author{
PRONAFCAP and its pedagogical practice, in beneficiary educational institutions, Puno region \\ PRONAFCAP e sua prática pedagógica, em instituições de ensino beneficiárias, região de Puno
}

\section{ARTÍCULO ORIGINAL}

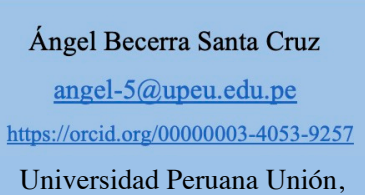

Universidad Peruana Unión, Lima - Perú
María Elizabeth Minaya Herrera

mariaminaya@upeu.edu.pe

https://orcid.org/00000003-3682-5664

Universidad Peruana Unión, Lima - Perú
Salomón Vásquez Villanueva

salomonv@upeu.edu.pe

https://orcid.org/0000-00018824-6176

Universidad Peruana Unión, Lima - Perú
Rubén Huanca Callasaca

rh18087@gmail.com

https://orcid.org/0000-00032861-7822

Universidad Peruana Unión, Lima - Perú

Recibido 18 de Enero 2021 | Arbitrado y aceptado 10 de Marzo 2021 | Publicado en 05 Junio 2021

\section{RESUMEN}

El objetivo general del presente trabajo es determinar el impacto del PRONAFCAPUPeU,

especialización, sobre la mejora de la práctica pedagógica de los docentes de Historia, Geografía y Economía de las instituciones beneficiadas de la región Puno 2012-2013. Se ha trabajado con 38 docentes participantes, constituidos en el grupo experimental, con quienes se ha desarrollado el programa y a quienes se ha aplicado las pruebas de entrada y prueba de salida. Los análisis de las tablas y los estadísticos descriptivos muestran que existe una diferencia significativa entre los resultados obtenidos en la prueba de entrada y en la prueba final, siendo mayor los resultados de la prueba final. Se concluye que el PRONAFCAPUPeU tuvo un impacto muy significativo sobre la mejora de la práctica pedagógica de los docentes $\mathrm{y}$ especialidades referidas.

Palabras claves: PRONAFCAP, práctica pedagógica profesional.

\section{ABSTRACT}

The general objective of the present work is to determine the impact of PRONAFCAP-UPeU, specialization, on the improvement of the pedagogical practice of the teachers of History, Geography and Economics of the benefited institutions of the Puno region 2012-2013. We have worked with 38 participating teachers, constituted in the experimental group, with whom the program has been developed and to whom the entrance and exit tests have been applied. The analyzes of the tables and the descriptive statistics show that there is a significant difference between the results obtained in the entrance test and in the final test, the results of the final test being higher. It is concluded that the PRONAFCAP-UPeU had a very significant impact on the improvement of the pedagogical practice of teachers and referred specialties.

Keywords: PRONAFCAP, professional pedagogical practice

\section{RESUMO}

O objetivo geral deste trabalho é determinar o impacto do PRONAFCAPUPeU, especialização, na melhoria da prática pedagógica dos professores de História, Geografia e Economia das instituições beneficiárias da região de Puno 2012-2013. Trabalhamos com 38 professores participantes, constituídos no grupo experimental, com os quais o programa foi desenvolvido e aos quais foram aplicados os testes de entrada e saída. As análises das tabelas e da estatística descritiva mostram que existe uma diferença significativa entre os resultados obtidos na prova de entrada e na prova final, sendo os resultados da prova final superiores. Conclui-se que o PRONAFCAP-UPeU teve um impacto muito significativo na melhoria da prática pedagógica dos professores e especialidades afins.

Palavras-chave: PRONAFCAP, prática pedagógica profissional. 


\section{INTRODUCCIÓN}

Los programas de capacitación, con sus aciertos y desaciertos, tienen una larga data histórica, en los países de diferente habla y de diversas regiones, respondiendo a políticas nacionales e internacionales, con prepuestos de los propios Estados y de los bancos internacionales. Se han realizado y desarrollado muchos.

En el Perú, el Programa de Mejoramiento de la Calidad de la Educación Peruana (MECEP), tuvo uno de sus subprogramas, denominado Plan Nacional de Capacitación Docente (PLANCAD), el cual tuvo éxito y recepción en el sector de la ecuación peruana, aplicado primero en Lima, luego en sus provincias y, posteriormente, se desarrolló a nivel nacional (de Belaunde, González, and Eguren, 2013). La aparición del PRONAFCAP significó la oportunidad para los docentes de los niveles: inicial, primaria y secundaria; primero fue la oportunidad para los docentes de primaria, luego para los docentes de inicial y posteriormente para los docentes del nivel de educación secundaria (Chacón Acosta, Montalván Acosta y Reátegui Acosta, 2013). En este contexto, es muy necesaria y muy importante la evaluación que se realiza sobre los programas, los cuales preparan a los profesionales de las diversas carrereas profesionales, con el propósito de mejorar la calidad del proceso enseñanza aprendizaje, mucho más en las zonas rurales (Monteza, 2018)

En este sentido y sobre estos programas, en el presente estudio se pretendió trabajar el objetivo: determinar el impacto profesional que genera el PRONAFCAP-UPeUespecialización en la mejora de la práctica pedagógica de los docentes de Historia, Geografía y Economía de las instituciones educativas beneficiadas de la región Puno 2012-2013.

\section{Importancia del PRONAFCAP}

La importancia y la influencia del PRONAFCAP quedaron a la vista de los gestores y de los beneficiarios del programa. En realidad, el programa permitió el desarrollo de la gestión educativa, específicamente de la gestión pedagógica; de manera singular, en la planificación, la ejecución, y la evaluación, fueron desarrolladas estas tres dimensiones de la gestión pedagógica (Simeón Aguirre, 2013). Por su parte, Chacón Acosta et al. 
(2013) destacan la importancia del programa subrayando que ha mejorado significativamente el desempeño de los docentes. De acuerdo con Guzmán Larrea (2013), las estrategias didácticas, en el contexto del PRONAFCAP, fomentan y mejoran las habilidades del pensamiento crítico, desarrollan las capacidades, la interiorización de los valores, el cambio o refuerzo de las actitudes. La formación docente, qué significa, qué concepción se tiene sobre la misma. Para

Rodriguez Manrique (2010),

La formación docente representa un proceso activo, autónomo e independiente de conocimiento e investigación. Se estructura sobre bases teóricas, sociales, políticas y está acondicionado a la idea de la escuela, enseñanza, innovación, investigación y currículo. Esto implica el análisis y reflexión sobre el significado conceptual de la formación en todos los niveles (p. 15) En el Perú y los países sudamericanos, los respectivos ministerios de educación, son los encargados de administrar y gestionar la educación y sus políticas, de acuerdo con las leyes, los decretos, las directivas y los manuales de organización y funciones. En el Perú, el Ministerio de Educación “dirige la política sectorial en educación, cultura, ciencia y tecnología, deporte y recreación, en concordancia con la política general del Estado y los planes del desarrollo nacional” (Marqués Molías and Gisbert Cervera, 2011).

El Programa Nacional de Formación y Capacitación Permanente (PRONAFCAP) fue implementado en el Perú, desde el año 2007, administrado y referenciado por el Ministerio de Educación. Los beneficiarios fueron los docentes del nivel básico regular. Este programa responde a lineamientos políticos, educativos, pedagógicos, económicos, evaluativos. De manera sustantiva, la evaluación y las estrategias de formación. La responsabilidad la tuvieron los institutos pedagógicos y las universidades mediante sus facultades y programas de educación, del sector nacional; sin embargo, el año siguiente (2008) fueron convocadas las universidades privadas.

Por su parte, Rodríguez Manrique (2010) aborda el PRONAFCAP, subrayando los antecedentes históricos, lineamientos políticos, pedagógicos, las estadísticas técnicas y económicas y la evaluación de dicho programa. La capacitación de los docentes, en el Perú, siempre se ha constituido en un eje político, cuyo interés es público, propiciando la innovación y los cambios de la gestión educativa, la implementación de nuevas dinámicas 
y técnicas orientadas a mejorar la calidad educativa nacional, cuyos beneficiarios son los docentes en ejercicio, quienes, por extensión y resultados de la capacitación, beneficiarán a los estudiantes y sus aprendizajes. Según Vilca (2020), el PRONAFCAP ha mejorado del desarrollo pedagógico de los docentes, específicamente mejorando la formación ciudadana y cívica, cuya experiencia se realizó en la región Puno, por los años 2012-2013.

Contexto legal

El Programa se sustenta en las siguientes normas legales. Ley de la carrera Pública Magisterial No 29062 y DS 003- 2008- ED que aprueba el Reglamento de la Ley N²9062. Decreto Supremo 002- 2007 que señala que la capacitación docente es acción prioritaria del Estado. Decreto Supremo Nº07- 2007-ED que crea el Programa Nacional de Formación y Capacitación Permanente, responsable de desarrollar las acciones conducentes a mejorar la formación en servicio de los profesores de la Instituciones educativas públicas a nivel nacional. Diseño Curricular Nacional de la Educación Básica Regular. R.M.N 0440- 2008ED. RM Nº017-2010-ED que aprueba el documento de política educativa del Programa Nacional de Formación y Capacitación Permanente2010 (PRONAFCAP 2010) "Mejores maestros, mejores alumnos"

\section{Marco histórico}

El año 1972, durante el gobierno militar de Juan Velasco Alvarado, aparece la iniciativa de las capacitaciones para los docentes, generada mediante la denominación de Reforma Educativa Peruana, con la orientación de construir un perfil intelectual del docente, cuya responsabilidad, entre otros, la tenía la los Núcleos Educativos Comunales (NEC). El año 1982, se fomentó el Programa Nacional de Revisión y Experimentación de los Programas de Estudio en Educación Inicial, Primaria y Secundaria; sin dejar al margen los Programas no Escolarizados de Educación Inicial (PRONOEI); entre 1995 y 2001, se desarrolló el Plan Nacional de Capacitación Docente (PLANCAD). También el Sindicato Único de

Trabajadores en la Educación del Perú (SUTEP), por el año 2005, tuvo parte activa en este quehacer (Rodríguez Manrique, 2007). 


\section{METODOLOGÍA}

\section{Tipo y diseño de estudio}

El estudio corresponde a una investigación experimental, fue manipulada la variable dependiente: práctica pedagógica, mediante la aplicación del Programa Nacional de Formación y Capacitación Permanente.

De acuerdo con Hernández Sampieri, Fernández Collado y Baptista Lucio (2014), el estudio corresponde a un pre-experimento, con diseño de pre prueba y pos prueba con un solo grupo, porque fue el único de especialización de docentes, en historia, geografía y economía durante los años 2012-2013.

\section{Delimitación espacial y temporal}

La investigación se realizó con docentes del sector público de la especialización de historia, geografía y economía de la región Puno, para mejorar su práctica pedagógica con sus estudiantes.

\section{Participantes}

El total de los participantes registrados fueron 53 docentes de historia, geografía y economía, entre varones y mujeres; sin embargo, solamente participaron 38 .

Técnicas de recolección de datos

Se utilizaron pruebas de entrada y salida, aplicadas respectivamente antes y después de la aplicación del programa, cuyas pruebas fueron validadas por especialistas del Ministerio de Educación y también por los especialistas de la Universidad Peruana Unión, sede Lima.

Plan de tratamiento de datos

Para ejecutar el tratamiento de las evaluaciones hechas a los estudiantes, se ha realizado el siguiente procedimiento: aplicación o toma de la prueba de entrada, calificaciones de la prueba, desarrollo del programa, aplicación la prueba de salida, tabulación de los resultados de las pruebas, registro de resultados mediante el estadístico SPSS 22, presentación de los datos en tablas estadísticas, lectura e interpretación de los datos. 


\section{RESULTADOS}

\section{Prueba de hipótesis}

Como las pruebas de normalidad nos muestra una distribución normal para ambos test entonces procedemos a la prueba de hipótesis aplicando la prueba paramétrica de $t$ de Student para una muestra con datos apareados.

Prueba de t de Student "pareado"

$$
T_{e x p}=\frac{\bar{d}-\Delta}{\frac{1}{\sqrt{n}} \hat{\mathcal{S}}_{d}} \sim \mathbf{t}_{n-1}
$$

Como se tienen dos pruebas, la de pretest y postest, tomado a una muestra en de 38 profesores quienes terminaron el programa de capacitación, y se obtuvieron sus correspondientes promedios y se esperó que la media del postest sea mayor respecto a la media del pretest a un nivel de confianza del 95\%, se tiene que en ambos casos se trata de una muestra cuyo comportamiento es del tipo normal, planteamos las hipótesis siguientes:

Para el componente dominio de los contenidos del área curricular

Ho: El impacto profesional que genera el PRONAFCAP-UPeU- especialización en el dominio de los contenidos del área curricular no es significativo en los docentes de Historia, Geografía y Economía de las instituciones educativas beneficiadas de la región Puno 2012-2013 ( $\mu 2 \leq \mu 1$, es decir: $\mu 2-\mu 1 \leq 0)$.

H1: El impacto profesional que genera el PRONAFCAP-UPeU- especialización

en el dominio de los contenidos del área curricular es significativo en los docentes de Historia, Geografía y Economía de las instituciones educativas beneficiadas de la región Puno 2012-2013 ( $\mu 2>\mu 1$; es decir: $\mu 2-\mu 1>0)$. 
Tabla 1.

Estadísticas de muestras emparejadas componente: Dominio de los contenidos del área curricular comparación de medias.

Media de

Desviación error

Media N estándar estándar

$\begin{array}{llllll}\text { Evaluación salida } \quad 16,39 & 38 & 2,871 & \text { 466 Evaluación entrada } \quad 10,71 \quad 38\end{array}$ $2,799,454$

Evalsalida - Evalentrada $\quad 5,684 \quad 38 \quad 3,706,601$

Tabla 2.

Prueba de muestras emparejadas componente: Dominio de los contenidos del área curricular

\section{Diferencias emparejadas}

$95 \%$ de intervalo de

confianza de la diferencia

Sig.

Superior $\quad \mathrm{t} \quad \mathrm{gl}$ (bilateral)

\begin{tabular}{lllll}
\hline Evalsalida - Evalentrada & 6,902 & 9,455 & 37 &, 000
\end{tabular}


El valor de significancia en la tabla, está dado por:

Sig. $\quad=0.000$

Por lo tanto:

Sig. $\quad<\alpha$

Luego entonces decimos que $0.000<0.05$. Por consiguiente, aceptamos la hipótesis alternativa con un nivel de significancia menor al 5\% o igual a cero, la cual confirma la hipótesis planteada, indicándonos que El impacto profesional que genera el PronafcapUPeU- especialización en el dominio de los contenidos del área curricular es significativo en los docentes.

Para el componente Estrategias Metodológicas Especificas del área.

Ho: El impacto profesional que genera el Pronafcap-UPeU- especialización en el dominio de estrategias metodológicas específicas del área no es significativo en los docentes.

H1: El impacto profesional que genera el Pronafcap-UPeU- especialización en el dominio de estrategias metodológicas específicas del área es significativo en los docentes.

Tabla 3.

Estadísticas de muestras emparejadas componente: Estrategias Metodológicas Especificas del área comparación de medias. 


\begin{tabular}{lcclc} 
& Media & $\mathrm{N}$ & $\begin{array}{l}\text { estándar } \\
\end{array}$ & estándar \\
\hline Evaluación salida & 12,63 & 38 & 1,667 &, 270 \\
Evaluación entrada & 8,84 & 38 & 2,354 &, 382 \\
Eval salida - Eval entrada & 3,789 & 38 & 2,462 &, 399 \\
\hline
\end{tabular}

Tabla 4.

Prueba de muestras emparejadas Componente: Estrategias Metodológicas Especificas del área.

Diferencias emparejadas

$95 \%$ de intervalo de

confianza de la diferencia

Sig.

Superior $\quad \mathrm{t} \quad \mathrm{gl}$ (bilateral)

\begin{tabular}{llrll}
\hline EvalSalida - Evalentrada & 4,599 & 9,487 & 37 &, 000
\end{tabular}

El valor de significancia en la tabla, está dado por:

Sig. $=0.000$

Por lo tanto:

Sig. $\quad<\alpha$ 
Luego entonces decimos que $0.000<0.05$. Por consiguiente, aceptamos la hipótesis alternativa con un nivel de significancia menor al 5\% o igual a cero, la cual confirma la hipótesis planteada, indicándonos que el impacto profesional que genera el PRONAFCAP-UPeU, en el dominio de estrategias metodológicas específicas del área es significativo en los docentes.

Para el componente investigación

Ho: El impacto profesional que genera el PRONAFCAP-UPeU- especialización en investigación no es significativo en los docentes.

H1: El impacto profesional que genera el PRONAFCAP-UPeU- especialización en investigación es significativo en los docentes.

Tabla 5.

Estadisticas de muestras emparejadas componente: Investigación

\begin{tabular}{|c|c|c|c|c|}
\hline & Media & $\mathrm{N}$ & $\begin{array}{l}\text { Desviación } \\
\text { estándar }\end{array}$ & $\begin{array}{l}\text { Media de } \\
\text { error estándar }\end{array}$ \\
\hline Evaluación salida & 13,66 & 38 & 2,602 & ,422 \\
\hline Evaluación entrada & 7,82 & 38 & 2,369 & ,384 \\
\hline Evalsalida - Evalentrada & 5,842 & 38 & 3,673 & ,596 \\
\hline
\end{tabular}

Para el PRONAFCAP en general 
Ho: El impacto profesional que genera el PRONAFCAP-UPeU- especialización en la mejora de la práctica pedagógica, no es significativo en los docentes.

H1: El impacto profesional que genera el PRONAFCAP-UPeU- especialización en la mejora de la práctica pedagógica, es significativo en los docentes.

Tabla 6.

Estadísticas de muestras emparejadas Programa de Capacitación Nacional

\section{Docente}

\begin{tabular}{lllll}
\hline & & & & Media de \\
& Media & $\mathrm{N}$ & $\begin{array}{l}\text { Desviación } \\
\text { estándar }\end{array}$ & $\begin{array}{l}\text { error } \\
\text { estándar }\end{array}$ \\
& 42,68 & 38 & 6,316 & 1,025 \\
\hline Evaluación salida & 27,37 & 38 & 6,175 & 1,002 \\
Evaluación entrada & 15,316 & 38 & 8,061 & 1,308 \\
Evalsalida - Evalentrada & & & & \\
\hline
\end{tabular}

\section{DISCUSIONES Y CONCLUSIONES}

Chacón Acosta et al. (2013) realizaron una investigación sobre el PRONAFCAP, cuyo estudio tuvo el objetivo de determinar la eficacia de dicho programa, dicha investigación es básica, descriptiva, explicativa, dentro de los parámetros de un estudio de enfoque cuantitativo, con la participación de 140 profesoras y 50 directoras, elegidas en forma aleatoria, cuyos cuestionarios fueron validados mediante los jueces; además, se estableció el nivel de confiabilidad estadística de 0.95 y de 0.98 , para las dimensiones eficacia del programa y desempeño docente, respectivamente. Los resultados muestran que el programa fue eficaz, expresado al $86.5 \%$.

Guzmán Larrea (2013), por su lado, desarrolla su investigación, con el objetivo de mejorar la práctica pedagógica, en el ámbito del PRONAFCAP, fomentar y desarrollar 
las habilidades del pensamiento crítico; para lograr el objetivo, se realizó una investigación acción pedagógica, usando el método de indagación, que ha permitido recabar y discriminar la información pertinente; en este sentido, los resultados permiten sostener que la práctica pedagógica enriquece las habilidades del pensamiento crítico de los estudiantes, quienes pierden el miedo, los temores y adquieren seguridad en su intervención. En la opinión y los estudios, de Zapata y Blanco (2012), las prácticas de la enseñanza cumplen una función muy importante, fundamental, sobre la formación de los futuros profesores, quienes vinculan la teoría con la práctica educativa, generando contacto directo con los estudiantes durante el proceso enseñanza aprendizaje, en el espacio denominado aula.

En realidad, en este espacio de la discusión, se afirma que los estudios e investigaciones consultados son, en su mayoría, bastante disímiles, heterogéneos, con escasas denominaciones comunes; por ejemplo, en la metodología, en los diseños, en los objetivos, en los propósitos e intenciones, en los niveles educativos, en las especialidades. Sin embargo, se aprecia que el PRONAFCAP, gestionado por el Ministerio de Educación en el Perú, tuvo sus grandes aciertos. Mejoró la aplicación de las estrategias pedagógicas por parte de los docentes, permitiendo que los estudiantes sean más activos en la generación o construcción de sus propios aprendizajes significativos, despertó la participación activa mediante el cultivo de la comunicación horizontal, llevando hacia el generación y desarrollo de sus habilidades sociales y comunicativas. En el escenario académico, se percibió apretura, diálogo, iniciativa; se exterminó, en cierta medida, la apatía, la indiferencia, la negación, la inhibición, en las dos partes: estudiantes y profesores.

En términos generales, en concordancia con los resultados obtenidos y habiendo efectuado el análisis en ellos acerca del impacto del PRONAFCAP-UPeUespecialización en la mejora de la práctica pedagógica de los docentes de Historia, Geografía y Economía de las instituciones educativas beneficiadas de la región Puno 2012-2013, me permiten formular las siguientes conclusiones:

El impacto profesional que genera el PRONAFCAP-UPeU- especialización en la mejora de la práctica pedagógica, es significativo en los docentes, porque la diferencia de medias es estadísticamente significativa de (15.316) con una t de Student de 11.712 con una significancia de 0.000 que es menor a 0.05 . 
El impacto profesional que genera el PRONAFCAP-UPeU- especialización en el dominio de los contenidos del área curricular es significativo en los docentes, la diferencia de medias es estadísticamente significativa de $(5,684)$ con una t de Student de 9,455 con una significancia de 0.000 que es menor a 0.05 .

El impacto profesional que genera el PRONAFCAP-UPeU- especialización en el dominio de estrategias metodológicas específicas del área es significativo en los docentes, la diferencia de medias es estadísticamente significativa de $(3,789)$ con una t de Student de 9,487 con una significancia de 0.000 que es menor a 0.05 .

El impacto profesional que genera el PRONAFCAP-UPeU- especialización en investigación es significativo en los docentes, la diferencia de medias es estadísticamente significativa de $(5,842)$ con una t de Student de 9,806 con una significancia de 0.000 que es menor a 0.05 .

Se habla de la importancia y de la influencia del PRONAFCAP. Al respecto, Simeón Aguirre (2013) realiza una investigación en un estudio de casos, mediante una metodología cualitativa, en 10 docentes de educación primaria, con la "necesidad de mayor interpretación valorativa, explicativa e inductiva que permita comprender los cambios y transformaciones" (p.x). En esta investigación se sostiene que el PRONAFCAP dejó una influencia positiva sobre el desarrollo de la gestión pedagógica, logrando la optimización de la gestión pedagógica, en sus dimensiones:

“planificación, ejecución y evaluación curricular”.

\section{REFERENCIAS}

Chacón Acosta, E., Montalván Acosta, G., and Reátegui Acosta, S. (2013). Eficacia del PRONAFCAP 2010 en la mejora del nivel de desempeño de las docentes de educación inicial de la UGEL 05, San Juan de Lurigancho, 2011. [Tesis de maestría, Universidad César Vallejo].

Retrieved from

http://repositorio.ucv.edu.pe/bitstream/handle/20.500.12692/9722/Chacon_AE

$$
\text { _Montalvan_AG.pdf?sequence }=1 \& \text { isAllowed }=\mathrm{y}
$$

de Belaunde, C., González, N., and Eguren, M. (2013). ¿Lección para el maestro? 
La experiencia del Plan Nacional de Capacitación Docente- PLANCAD. Lima, Perú: Edición digital del Instituto de Estudios Peruanos. Retrieved from https://repositorio.iep.org.pe/bitstream/IEP/951/2/documentodetrabajo188.pdf

Guzmán Larrea, R. E. (2013). Estrategias didácticas que fomentan habilidades del pensamiento crítico. UCV-HACER. Revista de Investigación Y Cultura, 2(1), 178-188. Retrieved from https://www.redalyc.org/pdf/5217/521752180021.pdf

Hernández, R., Fernández, C., and Baptista, M. del P. (2014). Metodología de la Investigación (Sexta edic). México, D.F: McGraw-Hill Interamericana

Editores, S.A. de C.V.

Marqués Molías, L., and Gisbert Cervera, M. (2011). Importancia de las competencias tic en el marco del PRONAFCAP. EDUTEC. Revista Electrónica de Tecnología Educativa, (36), 1-15. Retrieved from https://www.edutec.es/revista/index.php/edutece/article/view/403/139

Monteza, G. I. (2018). Evaluación del componente formativo de un programa para profesionales en aula. [Tesis de maestría, Pontificia Universidad Católica del Perú]. Retrieved from

http://tesis.pucp.edu.pe/repositorio/bitstream/handle/20.500.12404/14200/MO

NTEZA_FERNÁNDEZ_GABRIELA_INÉS11.pdf?sequence $=1 \&$ isAllowed $=\mathrm{y}$

Rodríguez Manrique, C. P. (2007). El Programa Nacional de Formación y Capacitación Permanente (Pronafcap ). Educación, XIX(37), 87-103. Retrieved from http://disde.minedu.gob.pe/bitstream/handle/123456789/1226/759. El

Programa Nacional de Formación y Capacitación Permanente \%28Pronafcap $\% 29 . . p d f ?$ sequence $=1 \&$ isAllowed $=\mathrm{y}$

Rodríguez Manrique, C. P. (2010). El programa de formación docente 
PRONAFCAP 2008 (CISE-PUCP): una mirada a los procesos de planificación y evaluación. [Tesis de maestría, Pontificia Universidad Católica del Perú]. Retrieved from http://tesis.pucp.edu.pe/repositorio/handle/20.500.12404/1227

Simeón Aguirre, A. M. (2013). La influencia del PRONAFCAP en la gestión pedagógica de la I.E “Fe y Alegría No 53" UGEL No6 Ate-Vitarte 2013. [Tesis de maestría, $\begin{array}{llll}\text { Universidad } & \text { César } & \text { Vallejo]. } & \text { Retrieved }\end{array}$ http://repositorio.ucv.edu.pe/bitstream/handle/20.500.12692/13426/Simeón_A

AM.pdf?sequence=1

Vilca, J. N. (2020). Efecto del Pronafcap-UPeU en el perfeccionamiento del desarrollo pedagógico de los docentes de Formación Ciudadana y Cívica de la

Región Puno 2012-2013. [Tesis de grado maestro, Universidad Peruana

Unión].

Retrieved

from

https://repositorio.upeu.edu.pe/bitstream/handle/UPEU/4184/Julian_Tesis_Mae stro_2020.pdf?sequence $=1 \&$ isAllowed $=\mathrm{y}$

Zapata, M. A., and Blanco, L. J. (2012). Las prácticas de enseñanza. Formación inicial del profesorado de Matemáticas. Educación Matemática, 25(1), 155-

159. Retrieved from http://www.scielo.org.mx/pdf/ed/v25n1/v25n1a7.pdf 